\title{
THE ALARM DISTRESS BABY SCALE IN A LONGITUDINAL PORTUGUESE STUDY
} REANALYZED WITH ATTACHMENT DATA

\author{
RAQUEL COSTA \\ Universidade Europeial Laureate International Universities \\ BÁRBARA FIGUEIREDO \\ University of Minho
}

ABSTRACT: The relationship established between the infant and the caregiver is central to both parents and infants, and provides one of the most important environments in wich children develop. This study aimed to assess the effect of infant's psychophysiological functioning early in life on the quality of mother-infant interaction and on later attachment, and to explore the mediation effects of the quality of mother-infant interaction on the association between the infant's psychophysiological functioning and attachment security. A longitudinal prospective design was conducted with 94 infants and their mothers. Eight-week-old infants were assessed with the Neonatal Behavioral Assessment Scale (T.B. Brazelton \& J.K. Nugent, 1995) and the Alarm Distress Baby Scale (A. Guedeney \& J. Fermanian, 2001). At 8 to 12 weeks of age, cortisol levels were measured both before and after routine inoculation. Mother-infant interaction was evaluated at 12 to 16 weeks, using the Global Rating Scales (L. Murray, A. Fiori-Cowley, R. Hooper, \& P. Cooper, 1996). The Strange Situation procedure (M. Ainsworth, M. Blehar, E. Waters, \& S. Wall, 1978) was performed at 12 months. The overall quality of mother-infant interaction mediates the relation between infant's behavioral and physiological profile and infant attachment: The probability of been securely attached increased with good mother behavior and with good overall interaction. The co-construction of the mother-infant relationship depends on the infant characteristics and on patterns of interaction.

Abstracts translated in Spanish, French, German, and Japanese can be found on the abstract page of each article on Wiley Online Library at http://wileyonlinelibrary.com/journal/imhj.

The relationship established between the infant and the caregiver is the earliest and closest among the many relationships that individuals experience throughout their life. These interactions are central to the lives of both parents and infants, and provide one of the most important environments in which children develop as individuals and as members of their culture (Russell, Mize, \& Bissaker, 2002). When discussing parent-child relationships, attention must be directed to the individuals as participants in the relationship, to the interpersonal aspects of the relationship, and to the broader social context and systems that influence parent-child relationships. Parent-child relationships are complex and multidimensional. They vary over time, differ from the perspective of the parent and of the child, and differ from one situation to another, and so on. Interaction problems at this time are associated with later developmental difficulties and attachment organization (Evans \& Porter, 2009).

Direct correspondence to: Raquel Alexandria Costa, ISLA Campus Lisboa/Laureate International Universities, Quinta do Bom Nome, Estrada da Correia, no 53, Lisboa, Portugal; e-mail: raquel.costa@1x.isla.pt.
The idea that both mother and infant characteristics influence the quality of their behavior in the interaction is consistent with a transactional model of development (Bell, 1974; Sameroff, 1975). From the transactional perspective, infants' and caretakers' characteristics exert a mutual and reciprocal influence, leading to unique patterns of behavior. Therefore, differences in the quality of attachment relationships arise after a history of infant-caregiver interactions (Weinfield, Sroufe, Egeland, \& Carlson, 1999). On the construction of this interaction background, both infant characteristics and maternal characteristics play a central role by influencing the interpretation and type of response to each other's behaviors (Sameroff, 2009).

Despite this, attachment theorists have conceived attachment as a relational construct independent of temperament while some temperament theorists have stated that attachment measures are alternative assessments of infant temperament (Rothbart \& Ahadi, 1994). Nonetheless, several investigators have proposed that infant characteristics might influence the quality of attachment or, at least, the behavior displayed in the Strange Situation. Furthermore, temperament theorists have agreed that caregiving practices 
can modify the expression of temperament, although they have not explained exactly how attachment might affect temperamental development. On the other hand, attachment theories have argued that the infant temperament variance is overshadowed by the more mature caregiver's success or failure in accommodating it (Goldsmith \& Alansky, 1987).

Bowlby (1969) argued that aspects of both the child's state and the novelty of the situation interfere with attachment behavior. Considering that proneness to distress influences the infants' state, then the nature of children's experience in situations relevant to attachment will differ (van den Boom, 1989). Temperament also might affect the development of attachment by mediating the course of mother-infant interaction (Goldsmith, Bradshaw, \& Rieser-Danner, 1986; Goldsmith \& Campos, 1986). Goldsmith, Bradshaw, and Rieser-Danner (1986) suggested that the attachment system activation, and especially proximity-seeking behavior, depends on infant fearfulness: In highly fearful child, a lower level of distress leads to fewer opportunities for experiencing the mother as a secure base for exploration. Other dimensions of temperament also have been associated to stranger sociability in several studies (Tavecchio \& van IJzendoorn, 1987). Activity level, adaptability, positive mood, and high threshold of response (Scarr \& Salapatek, 1970) as well as fear (Thompson \& Lamb, 1984) were related to stranger sociability. These studies have provided some evidence on the role of infant's individual differences very early in life for the development of attachment relationships.

On the other hand, evidence about the association between maternal variables measured during mother-child interaction and security of attachment also has been well-documented. Mothers who were more sensitive to their infants' cues for proximity and contact early in the first year of life (Ainsworth, 1979, 1982; Ainsworth, Bell, \& Stayton, 1971, 1974; de Wolff \& van IJzendoorn, 1997; Finger, Hans, Bernstein, \& Cox, 2009; Grossmann et al., 1985; Moran, Forbes, Evans, Tarabulsy, \& Madigan, 2008), more responsive and encouraging in face-to-face interaction (Blehar, Lieberman, \& Ainsworth, 1977; Goldsmith \& Alansky, 1987; Isabella, Belsky, \& von Eye, 1989), more emotionally open (Pauli-Pott \& Mertesacker, 2009; Ziv, Aviezer, Gini, Sagi, \& Koren-Karie, 2000), and more sensitive to their infants in freeplay activities (Fuentes, Lopes dos Santos, Beeghly, \& Tronick, 2006) were more likely to have securely attached infants. Mothers of securely attached infants are more affectionate (Bates, Maslin, \& Frankel, 1985), gentler (Londerville \& Main, 1981), accepting (Main, Tomasini, \& Tolan, 1979), positive in their vocalizations (Roggman, Langlois, \& Hubbs-Tait, 1987), and show more positive affect (Malatesta, Culver, Tesman, \& Shepard, 1989), as compared to mothers of insecurely attached infants. Mothers of avoidant infants were characterized by overstimulation and intrusiveness while mothers of resistant infants were characterized by underinvolvement and unavailability (Isabella et al., 1989).

An essential issue is that there are multifactorial aspects, associated with the infant and the mother as well as with the interaction between them, involved in the development of attachment relationships. Bates, Maslin, and Frankel (1985) noted that infants perceived as outgoing and fearless and infants perceived by their mothers as having low interest in them maintained less contact during the reunion episodes of the Strange Situation. These associations may have a biological basis, although the cause may be due to patterns of parent-infant interaction. When parents are the source of information, we may wonder wether the perception of the child is due to the child itself, the parent's inexperience, or pressures arising from the parent's mental health (MacKenzie \& McDonough, 2009).

In 1989, van den Boom examined the links between neonatal irritability at Days 10 and 15 of life, the quality of mother-infant interaction at Month 6, and infant attachment assessed at 12 months. She found that neonatal irritability predicted later attachment classification, especially the avoidant category. Furthermore, mothers of irritable infants tended to develop a pattern of interaction characterized by a progressive underinvolvement and unresponsiveness with age. Looking at this data, van den Boom developed and implemented an intervention program to enhance maternal sensitive responsiveness with irritable infants. Infants in the experimental gourp were less likely to be categorized as insecurely attached at 12 months. van den Boom's studies are indicative of both the strength of biologically founded characteristics in predicting later attachment and the influence of maternal skills when training is added. They have illustrated that the interaction between the infant predisposition and mother behavior may develop into a trajectory of experience for the child, with important developmental outcomes (Rothbart \& Ahabi, 1994).

One of the most pressing issues in contemporary attachment theory is to describe complete causal pathways to explain wellreplicated correlations between early care and subsequent patterns of secure-base behavior. In this study, we analyzed the effect of infant's behavioral and physiological functioning early in life on the quality of mother-infant interaction and on later attachment. In addition, we have explored the mediation effects of motherinfant interaction on the association between infant's behavioral and physiological functioning and attachment security.

In a previous study (Costa \& Figueiredo, 2012), three groups of infants with three different behavioral and physiological profiles ("withdrawn," "extroverted," and "underaroused") at 2 months were identified. The identification of these profiles was determined according to the infants' neurobehavioral performance, social withdrawal, and neuroendocrine reactivity to inoculation. Their withdrawn infants showed severe signs of social withdrawal, poor neurobehavioral performance, and high neuroendocrine reactivity; extroverted infants showed practically no signs of social withdrawal, and had a good neurobehavioral performance and an average to high neuroendocrine reactivity; and underaroused infants showed some signs of social withdrawal, average neurobehavioral performance, and low neuroendocrine reactivity.

Bearing in mind that "it takes two to become attached" (van den Boom, 1997, p. 593), the study of both infant behavioral and physiological functioning and early mother-infant interaction associated with infant attachment is of great interest. The purpose of this study is to consider bidirectional effects on the dyadic system 
TABLE 1. Sociodemographic and Medical Data

\begin{tabular}{|c|c|c|c|c|c|}
\hline $\begin{array}{l}\text { Maternal and } \\
\text { Gestational Data }\end{array}$ & & $(\%)$ & Neonatal Data & & $(\%)$ \\
\hline \multirow[t]{2}{*}{ Maternal Age } & $\leq 20 \Lambda \leq 34$ & 96.0 & $\begin{array}{l}\text { Time of } \\
\text { Gestation }\end{array}$ & $<37$ & 7.2 \\
\hline & $>35$ & 4.0 & & $\begin{array}{l}\geq 37 \Lambda \leq 40 \\
>40\end{array}$ & $\begin{array}{l}82.1 \\
10.7\end{array}$ \\
\hline \multirow[t]{2}{*}{ Year of Education } & $<9$ & 23.0 & Sex & Female & 46.9 \\
\hline & $\geq 9$ & 77.0 & & Male & 53.1 \\
\hline \multirow[t]{2}{*}{ Marital Status } & Married & 81.0 & $\begin{array}{l}\text { Reanimation at } \\
\text { Birth }\end{array}$ & No & 94.6 \\
\hline & Cohabiting & 19.0 & & Yes & 5.4 \\
\hline \multirow[t]{2}{*}{ Parity } & Primiparous & 84.2 & Weight & $<2,500 \mathrm{~g}$ & 1.7 \\
\hline & Multiparous & 15.8 & & $\geq 2,500 \mathrm{~g}$ & 98.3 \\
\hline \multirow[t]{2}{*}{ Type of Gestation } & Normal & 80.4 & Ponderal Index & $<2.5$ & 13.5 \\
\hline & Risk & 19.6 & & $\geq 2.5$ & 86.5 \\
\hline \multirow[t]{2}{*}{ Type of Delivery } & Vaginal & 34.2 & $\begin{array}{l}\text { Apgar Index: } \\
1 \text { min }\end{array}$ & $<7$ & 3.8 \\
\hline & Cesarean & 65.8 & & $\geq 7$ & 96.2 \\
\hline \multirow[t]{3}{*}{ Type ofAnesthesia } & None & 2.6 & Type of feeding & Breast-Fed & 89.4 \\
\hline & Epidural & 86.8 & & Bottle-Fed & 10.6 \\
\hline & General & 10.5 & & & \\
\hline
\end{tabular}

and the way in which they contribute to the co-construction of the infant-mother relationship.

\section{METHOD}

\section{Sample}

The sample was composed of 94 infants. Most infants were born after a normal and full-term gestation. More than half were born through a cesarean section and had no need for reanimation. At birth, infants height ranged from 45.90 to $54.00 \mathrm{~cm}(M=49.44$, $S D=1.84)$, cephalic perimeter ranged from 31 to $37 \mathrm{~cm}(M=$ $34.60, S D=1.29)$, weight ranged from 2,450 to $4,055 \mathrm{~g}(M=3243$, $S D=424)$, ponderal index ranged from 2.24 to $3.29(M=2.71$, $S D=0.23)$, and Apgar scores ranged from 5 to $10(M=8.63$, $S D=0.91)$ at $1 \mathrm{~min}$ and from 8 to $10(M=9.76, S D=0.53)$ at 5 min (see Table 1).

\section{Procedures}

This research was conducted in the Primary Care Centers of Espinho and Santa Maria da Feira (Portugal) after the protocol was analyzed and approved by the ethical committee. Mothers were contacted when attending routine inoculation of their 1-month-old infant; $96 \%$ of the contacted mothers agreed to participate, $3 \%$ declined participation alleging lack of time, and $1 \%$ were not interested in participating. The exclusion criteria were not reading or writing Portuguese and/or multiple gestations. The aims and the procedures of the study were explained, and an informed consent was signed. All evaluation procedures were performed and videotaped either at home or at the Primary Care Center.
A sociodemographic questionnaire was completed on infants' medical data, and when the infant was 8 weeks old ( \pm 5 days), the Neonatal Behavioral Assessment Scale (NBAS; Brazelton \& Nugent, 1995) was performed and videotaped. This examination was conducted in a particular sequence by trained and reliable examiners midway between feedings in a quiet and semidarkened room with a temperature of 22 to $27^{\circ} \mathrm{C}$. The NBAS was scored immediately after being performed. At this time, the infant's social withdrawal behavior also was assessed using the Alarm Distress Baby Scale (ADBB; Guedeney \& Fermanian, 2001). The ADBB was scored by the researcher who had carried out the NBAS procedure. Between 8 and 12 weeks of life, a saliva sample was collected from the infant's mouth before (5-10 $\mathrm{min}$ ) and after (20-22 $\mathrm{min}$ ) routine inoculation. Mother-infant interaction was evaluated at 12 to 16 weeks, using the Global Rating Scales (GRS; Murray, Fiori-Cowley, Hooper, \& Cooper, 1996). The Strange Situation procedure was performed to assess infant attachment style between 12 and 14 months (Ainsworth et al., 1978).

\section{Measures}

Neonatal behavior. The NBAS (Brazelton \& Nugent, 1995) assesses the newborn's competencies across different developmental areas-autonomic, motor, states and social-and describes how these areas are integrated. The autonomic stability records signs of stress related to homeostatic adjustments of the central nervous system The motor factor measures motor performance and the quality of movement and tone. Range of state is a measure of infant arousal and state lability. The regulation of state reports the infant's ability to regulate his or her state in the presence of increasing levels of stimulation. The Orientation factor includes the ability to attend to visual and auditory stimuli and the quality of overall alertness in social interactions (Brazelton \& Nugent, 1995).

The scale, composed of 28 behavioral and 18 reflex items, is suitable for examining newborns and infants up to 2 months old and is based on three key assumptions: (a) Infants are highly competent when they are born, (b) infants "communicate" through their behavior, and (c) infants are social organisms. By the end of the assessment, the examiner has a behavioral "portrait" of the infant, describing his or her strengths, adaptive responses, and possible vulnerabilities. The 28 items of the NBAS are scored on a 9 point scale. For the NBAS total score, behavioral and reflexes items were recoded so that a better performance corresponds to higher score and were then added.

Social withdrawal. The ADBB (Guedeney \& Fermanian, 2001) consists of eight items to assess prolonged reaction of social withdrawal in infants. Items are rated from 0 to 4 (with low scores being optimal social behavior) on facial expression, eye contact, general level of activity, self-stimulation gestures, vocalizations, briskness of response to stimulation, relationship to the observer, and attractiveness to the observer. The ADBB total score derives from the sum of the eight items, and higher results represent more signs of social withdrawal. The cutoff point of 5 showed the best 
sensitivity (0.82) and specificity (0.78) to detect infants at risk (Guedeney \& Fermanian, 2001). Interrater reliability was calculated using the intraclass coefficient (.92). The Portuguese version of the scale has a reasonable internal consistency (Cronbach's $\alpha=$.60) (Figueiredo \& Costa, 2008).

Mother-infant interaction. The GRS (Murray, Fiori-Cowley et al., 1996) is a video-based assessment of the quality of mother-infant engagement that can be applied from 2 to 6 months' postpartum. The mother sat in front of the infant and was asked to play with him or her in any way they chose without the use of toys in a 5-min, face-to-face play session. A video camera was set up to film the event to obtain a full image of the infants' body, and the mother's full-face image also was filmed using a mirror placed adjacently to the infant. During a 5-min, video-recorded assessment of free play between mother and infant, the scales globally assess the quality of (a) maternal behavior, (b) infant behavior, and (c) overall interaction. Maternal behavior describes the degree to which a mother's behavior is appropriately adjusted to her infant. Mother's behavior was computed using the sum score of three subscales: (a) Good-poor-computed through the average score of five items (warm/positive vs. cold/hostile, accepting vs. rejecting, responsive vs. unresponsive, nondemanding vs. demanding, sensitive vs. insensitive), with a sum score near 5 rated as "good," and a sum score near 1 rated as "poor;" (b) Intrusiveremote-composed of four items (nonintrusive behavior vs. intrusive behavior, nonintrusive speech vs. intrusive speech, nonremote vs. remote, nonsilent vs. silent); (c) Depressive-computed through the average of four items (happy vs. sad, much energy vs. low energy, absorbed in the infant vs. self-absorbed, relaxed vs. tense), with the higher score indicating less depressive signs.

Infant behavior describes the infants' positive engagement in the interaction and behavior. Infant behavior was computed according to two subscales, describing the infants' positive engagement in the interaction, and behavior: (a) Good-poor-computed through the average of three items (attentive vs. avoidant, active communication vs. no active communication, positive vocalizations vs. no positive vocalizations), with a sum score near 5 rated as "good," and a sum score near 1 rated as "poor;" and (b) Inertfretful-composed of four items (engaged with the environment vs. self-absorbed, lively vs. inert, attentive vs. avoidant, happy vs. distressed, nonfretful vs. fretful).

The final dimension assesses the quality of the overall interaction between mother and infant; it rates the nature of the engagement between mother and infant and was computed through the sum score of the overall interaction items. A higher the punctuation corresponds to a better performance. The overall interaction was rated using one subscale: Good-poor composed of the average score of five items (smooth/easy vs. difficult, fun vs. serious, satisfying vs. unsatisfying, much engagement vs. no engagement, excited engagement vs. quiet engagement); a sum score of 5 is considered "good interaction," and near 1 is considered "poor interaction."
Infant attachment style. The Strange Situation was performed (Ainsworth et al., 1978) and videotaped when the infants were between 12 and 14 months of age. Two expert coders classified infants as secure, insecure-avoidant, or insecure-resistant, as described in Ainsworth et al. (1978). Raters agreed on major classifications in $97.6 \%$ of the cases; disagreements were resolved by conference. The distribution of attachment classifications was $(61.9 \%)$ secure, $(21.6 \%)$ insecure-resistant, and (16.5\%) insecure-avoidant. In this study, we considered the classification insecure (0) vs. secure (1).

\section{RESULTS}

Using NBAS and ADBB scores as well as the levels of cortisol reactivity to inoculation, three behavioral and physiological profiles were determined through cluster analysis- "withdrawn," "extroverted," and "underaroused" — and are described elsewhere (Costa \& Figueiredo, 2012).

A multivariate analysis of variance (MANOVA) followed by a univariate $F$ test and a Bonferoni post hoc test (Field, 2005) were performed to identify potential differences on the quality of mother-infant interaction according to the infant's behavioral and physiological profile after the validation of the assumptions. The validation of the assumption of homogeneity of variancescovariances using Box's () M test was guaranteed, $M=93.635$, $F(37,3349)=.957, p=.137$.

The MANOVA performed to identify potential differences in the quality of mother-infant interaction according to the infant's behavioral and physiological profile was significant, $\Lambda=.724$, $F(2,94)=2.634 ; p<.05$. Subsequent univariate analyses followed by Bonferroni post hoc test indicated a significant effect for infant behavior and overall interaction, but not for mother behavior (see Table 2). Withdrawn infants had lower scores for infant behavior, as compared to extroverted (CI 95\% $=-1.38,-.40$ ), $p<.05$, and underaroused (CI 95\% $=-2.68,-.26), p<.05$, infants. Withdrawn infants had lower scores on overall interaction, as compared to extroverted infants (CI 95\% $=-1.41,-.29), p<$ .05 .

To explore if the infant's behavioral and physiological profile was associated with the secure versus the insecure attachment classification, the chi-square test was used. Significant associations were found between the infant's behavioral and physiological profile and attachment security, $\chi^{2}=5.442, p<.05$. More than half of withdrawn infants, one third of underaroused infants, and only one fourth of extroverted infants were insecurely attached at 12 months (see Table 3).

A MANOVA followed by a univariate $F$ test and Bonferoni post hoc test (Field, 2005) were performed to identify potential differences in the quality of mother-infant interaction in infants with secure versus insecure attachment after the validation of the assumptions. The validation of the assumption of homogeneity of variances-covariances using the Box's M test was guaranteed, $M=$ $88.563 ; F(35,2769)=.995, p=.097$.

Regarding the quality of mother-infant interaction and infant attachment, the MANOVA was significant, $\Lambda=.724$, 
TABLE 2. Differences in the Quality of Mother-Infant Interaction in Three Groups of Infants With Different Psychophysiological Profiles

\begin{tabular}{|c|c|c|c|c|c|c|}
\hline & $\begin{array}{l}\text { Withdrawn (A) } \\
\begin{array}{c}(n=16) \\
M(S D)\end{array}\end{array}$ & $\begin{array}{c}\text { Extroverted (B) } \\
\quad(n=56) \\
M(S D)\end{array}$ & $\begin{array}{l}\text { Underaroused }(\mathrm{C}) \\
\qquad \begin{array}{c}(n=25) \\
M(S D)\end{array}\end{array}$ & $F$ & $p$ & \\
\hline \multicolumn{7}{|c|}{ Mother-Infant Interaction } \\
\hline Mother behavior & $4.03(.73)$ & $4.25(.38)$ & $4.41(.52)$ & 1.881 & .164 & \\
\hline Infant behavior & $2.97(.93)$ & $4.33(.65)$ & $3.61(.88)$ & 6.709 & .003 & A vs. B; A vs. C \\
\hline Overall interaction & $2.76(.68)$ & $4.28(.57)$ & $3.41(.44)$ & 4.965 & .011 & A vs. B \\
\hline
\end{tabular}

TABLE 3. Association Between Infant Behavioral and Physiological Profile and Attachment Classification and Differences in the Quality of Mother-Infant Interaction According to Attachment Classification

\begin{tabular}{lcccc}
\hline & $\begin{array}{c}\text { Insecure }(n=36) \\
(\%)\end{array}$ & $\begin{array}{c}\text { Secure }(n=58) \\
(\%)\end{array}$ & $\chi^{2}$ & $p$ \\
\hline Infant Profile & & & & \\
$\quad$ Withdrawn & 66.7 & 33.3 & 5.442 & .046 \\
$\quad$ Extroverted & 25.0 & 75.0 & & \\
Underaroused & 38.9 & 61.1 & & \\
& $M(S D)$ & $M(S D)$ & $F$ & $p$ \\
\hline Mother-Infant Interaction & & & & \\
$\quad$ Mother behavior & $51,67(5,12)$ & $58,78(3,73)$ & 4.982 & .037 \\
$\quad$ Infant behavior & $24,94(3,75)$ & $30,16(4,34)$ & 3.947 & .049 \\
$\quad$ Overall interaction & $15,56(4,23)$ & $20,94(3,12)$ & 4.987 & .041 \\
\hline
\end{tabular}

$F(2,94)=2.634, p<.05$. Subsequent univariate analyses revealed that mean scores for mother behavior, $F(1,94)=4.982$, $p<.05$, infant behavior, $F(1,94)=3.947, p<.05$, and overall interaction, $F(1,94)=4.987, p<.05$, were significantly higher in securely attached infants as compared to insecure attached infants (see Table 3).

\section{Test of Mediation Model}

To determine if the quality of mother-infant interaction mediated the effect of the infant's behavioral and physiological profile on attachment security, several regression analyses were performed (Tabachnick \& Fidell, 1996). In the first equation, the infant's behavioral and physiological profile was entered as an independent variable and the infant attachment as the criterion (dichotomous variable: $0=$ insecure, $1=$ secure). In the second equation, the infant's behavioral and physiological profile was entered as an independent variable and the quality of mother-infant interaction as the criterion. In the third equation, the quality of mother-infant interaction was entered as an independent variable and the infant attachment as the criterion (dichotomous variable: $0=$ insecure, $1=$ secure). The fourth equation was conducted with the infant's behavioral and physiological profile and the quality of motherinfant interaction as independent variables, and the infant attachment as the criterion (dichotomous variable: $0=$ insecure, $1=$ secure). A “. . .variable may be said to function as a mediator to the extent that it accounts for the relation between the predictor and the criterion" (Baron \& Kenny, 1986, pp. 1176).

To test if mother-infant interaction accounted for the relation between infant behavioral and physiological profile and attachment, we analyzed four conditions considered to be essential to show mediation (Baron \& Kenny, 1986).

- Variations in an infant's behavioral and physiological profile account for variations in infant attachment,

- variations in an infant's behavioral and physiological profile account for variations in the quality of mother-infant interaction,

- variations in the quality of mother-infant interaction account for variations in the infant attachment, and

- a previously significant relation between an infant's behavioral and physiological profile and infant attachment is significantly reduced or no longer significant when the quality of mother-infant interaction is added to the model. If Path $c$ is reduced to zero, then mother-infant interaction can be considered a single mediator whereas if Path $\mathrm{c}$ is not zero, multiple mediating factors may exist (Baron \& Kenny, 1986).

The first logistic regression, revealed that an infant's behavioral and physiological profile, $\chi^{2}$ wald $(2)=4.926$, $p<.05$, has a significant effect on the probability of having a secure attachment (see Table 4). According to the model, $G^{2}(6)=$ $5.319, p>.05, \chi^{2}=35.015, R^{2}{ }_{\mathrm{CS}}=.086, R^{2}{ }_{\mathrm{N}}=.118, R_{\mathrm{MF}}^{2}=$ .069 , being withdrawn decreases the probability of being securely attached to the mother while being underaroused decreases the probability of being securely attached to the mother, as compared to extroverted infants.

Three linear regression analyses were performed to test Path a1, a2, and a3, exploring if the infant's behavioral and physiological profile accounted for variations in mother behavior, infant behavior, and overall quality of interaction. The variation on an infant's behavioral and physiological profile did not account for variations on mother behavior, $F(2,94)=1.591, p>.05$, but it accounted for variations on infant behavior, $F(2,94)=23.247, p<.01$, and overall interaction, $F(2,94)=16.488, p<.05$ (see Table 5$)$.

This result excludes mother behavior in the interaction as a potential mediator variable of the relation between an infant's 
TABLE 4. Predicting Infant Attachment From Infant Behavioral and Physiological Profile and Quality of Mother-Infant Interaction

\begin{tabular}{|c|c|c|c|c|c|}
\hline Variable & $B$ & $S E$ & $\chi^{2}$ Wald $d f \quad p$ & $\operatorname{Exp}(B)$ & $5 \%$ \\
\hline \multicolumn{6}{|c|}{ Infant Psychophysiological Profile } \\
\hline Extroverted & & & 4.9262 .085 & 1.810 & \\
\hline Withdrawn & -1.792 & 2.816 & 4.8161 .028 & .167 & $(.034, .826)$ \\
\hline Underaroused & -.647 & 7.633 & 1.0441 .307 & .524 & $(.152,1.811)$ \\
\hline \multicolumn{6}{|l|}{ Mother-Infant Interaction } \\
\hline Mother Behavior & .334 & 4.114 & $8.518 \quad 1.004$ & 1.396 & $(1.116,1.747)$ \\
\hline Infant Behavior & .144 & 4.281 & .2631 .608 & .866 & $(.499,1.502)$ \\
\hline Overall Interaction & .737 & 7.381 & 3.9811 .058 & 1.190 & $(.974,4.484)$ \\
\hline \multicolumn{6}{|l|}{ Mediating Effect } \\
\hline \multicolumn{6}{|l|}{$\begin{array}{l}\text { Extroverted } \times \text { Overall } \\
\text { Interaction }\end{array}$} \\
\hline $\begin{array}{l}\text { Withdrawn } \times \text { Overall } \\
\text { Interaction }\end{array}$ & .709 & 9.582 & $1.483 \quad 1.223$ & 2.032 & $(6.49,6.358)$ \\
\hline $\begin{array}{l}\text { Underaroused } \times \text { Overall } \\
\text { Interaction }\end{array}$ & .038 & 8.498 & $.006 \quad 1.939$ & 1.039 & $(.391,2.758)$ \\
\hline
\end{tabular}

TABLE 5. Predicting the Quality of Mother-Infant Interaction From Infant Psychophysiological Profile

\begin{tabular}{lcccccc}
\hline & $R^{2}$ & $F$ & $p$ & $\beta$ & $t$ & $p$ \\
\hline Mother Behavior & & & & & & \\
$\quad$ Withdrawn & .033 & 1.591 & .209 & -.192 & -1.763 & .081 \\
$\quad$ Underaroused & & & & -.036 & -.328 & .744 \\
Infant Behavior & & & & & & \\
$\quad$ Withdrawn & .336 & 23.247 & .000 & -.610 & -6.771 & .000 \\
$\quad$ Underaroused & & & & -.135 & -1.497 & .138 \\
Overall Interaction & & & & & & \\
$\quad$ Withdrawn & .264 & 16.488 & .000 & -.540 & -5.693 & .000 \\
$\quad$ Underaroused & & & & -.112 & -1.184 & .239 \\
\hline
\end{tabular}

behavioral and physiological profile and infant attachment because Path a1 was not confirmed (see Table 5).

To test Path b1, b2, and b3, a logistic regression was perfomed for mother behavior, infant behavior, and overall interaction. Mother behavior $\left(\mathrm{b}_{\text {MotherBehavior }}(1)=.334, p<.05\right.$, Odds Ratio $=1.396$ ) had a significant effect on the probability of having a secure attachment (Path b1) while the overall quality of interaction $\left(\mathrm{b}_{\text {OveralInteraction }}(1)=.737, p<.06\right.$, Odds Ratio $=2.090$ ) had a marginally significant effect on the probability of having a secure attachment. According to the model, $G^{2}(3)=35.015, p<.001, \chi^{2}=29.088, R^{2}{ }_{\mathrm{CS}}=.504, R^{2}{ }_{\mathrm{N}}=.697$, $R_{\mathrm{MF}}^{2}=.537$, the probability of being securely attached increased with good mother behavior and with good overall interaction. In contrast, infant behavior did not have a statistically significant effect $\left(b_{\text {InfantBehav }}(1)=.144, p>.05\right.$, on the probability of having a secure attachment (see Table 3 ).

This result excludes infant behavior in the interaction as a potential mediator variable of the relation between infant behavioral and physiological profile and infant attachment because Path b2 was not confirmed (see Table 3).

We then analyzed if the previously significant relation between infant behavioral and physiological profile and infant attachment decreased or disappeared after adding the overall interaction to the model to test the mediation model.

The logistic regression revealed that the association between infant behavioral and physiological profile and infant attachment decreased, but did not disappear, when the overall interaction was added in the equation, $G^{2}(2)=1.603, p>.05, R^{2}{ }_{\mathrm{CS}}=.017, R^{2}{ }_{\mathrm{N}}=$ $.023, R_{\mathrm{MF}}^{2}=.013$ (see Table 3 ). The data thus met the requirements for mediation.

\section{DISCUSSION}

The infant behavioral and physiological functioning early in life has a significant effect on the probability of having a secure attachment. More than half of withdrawn infants at 3 months are insecurely attached at 12 months, almost half of underaroused infants are insecurely attached, and only one fourth of extroverted infants are insecurely attached. Withdrawn infants are characterized by their high social withdrawal and low neurobehavior performance while underaroused infants are mainly characterized by their low neuroendocrine reactivity. Compared to extroverted infants, the probability of being securely attached decreases in withdrawn infants and in underaroused infants. This result is concordant with the results of previous studies that have shown that neurobehavior difficulties, low social responsiveness, unexcitability, not liking to play with others, low orienting ability, and higher distress reactivity and difficulty are related to insecure attachments (Bates et al., 1985; Calkins \& Fox, 1992; Grossmann et al., 1985; Seifer, Schiller, Sameroff, Resnick, \& Riordan, 1996; Waters, Vaughn, \& Egeland, 1980). It is possible that early neonatal difficulties are the reflection of problems in integrative and adaptative mechanisms that still influence the infant's behavior later in life, namely social interaction behavior (Waters et al., 1980).

Regarding the quality of mother-infant interaction, the results show that mean scores for mother behavior, infant behavior, and overall interaction are higher in securely attached infants. Good mother behavior in the interaction is characterized by warmth, acceptance, responsiveness, and sensitiveness and had a significant effect on the probability of having a secure attachment. This study therefore provides evidence consistent with a transactional model of development regarding the fact that parent behaviors as well as infant behaviors influence the quality of interaction. This association between mother behavior and the overall pattern of interaction and later infant attachment corroborates the attachment theory that holds that attachment relationships develop within the context of infant-mother interactions (Ainsworth et al., 1978; Bowlby, 1969).

We also found that the overall quality of interaction characterized by smooth, fun, satisfying, and excited engagement had a marginally significant effect on the probability of being securely attached. The study of mother-infant relationship qualities is 
crucial for understanding the transactional processes that contribute to the formation of different developmental pathways.

The infant's behavioral and physiological profile predicts infant behavior in the interaction as well as the quality of overall interaction, but not maternal behavior in the interaction. Extroverted infants are characterized by their good psychological performance while withdrawn infants are characterized by their poor psychological performance, and that seems to be reflected in the quality of mother-infant interactions. The results show that the better the psychological performance, the better the overall mother-infant interaction. Nugent et al. (1993) also noted a significant relation between neonatal behavior and the quality of mother-infant interaction. Similar results were obtained by Murray, Stanley, Hooper, King, and Fiori-Cowley (1996), who reported that poor motor performance and high levels of infant irritability in the neonatal period predicted worse infant behavior in face-to-face interactions with the mother at 2 months' postpartum. This study highligts the idea that infant characteristics influence the quality of his or her behavior in the interaction, and this is consistent with a transactional model of development (Bell, 1974; Sameroff, 1975). From the transactional perspective, parent's and infant's characteristics exert a mutual effect in each other, with important influence on the quality of interaction and with potential to transform it, leading to unique patterns of behavior.

In addition, the relationship between infant behavioral and physiological profile and infant attachment is mediated by the quality of overall interaction. As such, the overall interaction seems to be the primary pathway by which the infant's behavioral and physiological profile might impact on later attachment. Bates et al. (1985) also reported a correlation between infant characteristics and later attachment, and concluded that the cause of the correlation may be due to processes of the interaction. Goldsmith and Alansky (1987) demonstrated that sensitive, responsive, maternal interaction predicted the security of attachment while infant proneness to distress predicted resistance in the Strange Situation. In 1989, van den Boom found that infant's irritability predicted later attachment classification, especially the avoidant category, and that mothers of irritable infants get progressively less involved and more unresponsive to the infant over time. She proved that intervention programs aimed at enhancing maternal sensitive responsiveness with irritable infants had positive effects on infant attachment to the mother (van den Boom, 1989).

In withdrawn infants, maternal behavior seems to be particularly relevant for the development of secure/insecure attachments. We may then conclude that maternal behavior might have a differential impact on infant development according to his or her preexisting unique characteristics. Considering that infant behavior early in life influences the caretaking environment, difficulties at this time limit the quality of the mother-infant interaction (Waters et al., 1980). Caregiver behavior also is a function of infant behavior, and as such, early difficulties can be expected to limit the quality of the caregiving environment. Nonetheless, when mothers are able to overcome difficulties in coordinating their behavior with the withdrawn infant's functioning, this seems to have a protective effect on infant development. A probable explanation is that these mothers can provide more positive interaction experiences in the day-to-day activities for their infants. This is an important cue for clinical practice, once early intervention programs can be developed for mothers of withdrawn infants to help them overcome the difficulties inherent in their infant's behavior.

This study presents some limitations, including the fact that the sample consisted of primarily White, adult mothers with a simple gestation; the generalization of results is limited to this population. In adition, no data were collected regarding mothers' psychosocial status that could interfere with their behavior in the interaction. The fact that the NBAS and the ADBB were assessed by the same researcher also might had caused some bias. Nonetheless, the results of this study suggest that infant contributions to the development of particular patterns of mother-infant interaction and later attachment begin soon after birth. It also alerts to the fact that neither infant functioning nor caregiver behavior can be disregarded since both contribute to the development of the dyadic system and the relationship. Future research should address this issue in a larger sample to analyze the differential impact of both infant characteristics and mother-infant interaction on insecure-avoidant, insecure-resistant, and disorganized infants. Additional evidence also would be usefully regarding the timing of both infant difficulties and interaction problems on developmental outcomes.

\section{REFERENCES}

Ainsworth, M. (1979). Attachment as related to mother-infant interaction. In J.S. Rosenblatt, R.A. Hide, C. Beer, \& M. Busnel (Eds.), Advances in the study of behavior (Vol. 9, pp. 2-49). New York: Academic Press.

Ainsworth, M. (1982). Attachment: Retrospect and prospect. In C.M. Parkes \& J. Stevenson-Hinde (Eds.), The place of attachment in human behavior (pp. 3-30). New York: Basic Books.

Ainsworth, M., Bell, S.M., \& Stayton, D. (1971). Individual differences in Strange Situation behavior of one-year-olds. In H.R. Schaffer (Ed.), The origins of human social relations (pp. 17-57). London: Academic Press.

Ainsworth, M., Bell, S.M., \& Stayton, D. (1974). Infant-mother attachment and social development. In M.P. Richards (Ed.), The introduction of the child into a social world (pp. 99-135). London: Cambridge University Press.

Ainsworth, M., Blehar, M., Waters, E., \& Wall, S. (1978). Patterns of attachment. Hillsdale, NJ: Erlbaum.

Atkinson, L., Goldberg, S., Raval, V., Pederson, D., Benoit, D., Moran, G. et al. (2005). On the relation between maternal state of mind and sensitivity in the prediction of infant attachment security. Developmental Psychology, 41(1), 42-53.

Baron, R.M., \& Kenny, D.A. (1986). The moderator-mediator variable distinction in social psychological research: Conceptual, strategic, and statistical considerations. Journal of Personality and Social Psychology, 51, 1173-1182. 
Bates, J.E., Maslin, C.A., \& Frankel, K.A. (1985). Attachment security, mother-child interaction, and temperament as predictors of behaviorproblem ratings at age three years. In I. Bremerton \& E. Waters (Eds.), Growing points of attachment theory and research. Monographs of the Society for Research in Child Development, 50(1-2, Serial No. 209).

Bell, R.Q. (1974). Contributions of human infants to caregiving and social interaction. In M. Lewis \& L. Rosenblum (Eds.), The effect of the infant on its caregiver (pp. 1-19). New York: Wiley.

Blehar, M.C., Lieberman, A.F., \& Ainsworth, M. (1977). Early face-toface interaction and its relation to later infant-mother attachment. Child Development, 48, 182-194.

Bowlby, J. (1958). The nature of the child's tie to its mother. International Journal of Psychoanalysis, 39, 350-373.

Bowlby, J. (1969). Attachment and loss (Vol. 1): Attachment. New York: Basic Books.

De Wolff, M.S., \& van IJzendoorn, M.H. (1997). Sensitivity and attachment: A meta-analysis on parental antecedents of infant attachment. Child Development, 68, 571-591.

Evans, C.A., \& Porter, C.L. (2009). The emergence of mother-infant co-regulation during the first year: Links to infants' developmental status and attachment. Infant Behavior \& Development, 32(2), $147-158$.

Field, A. (2005). Discovering statistics using SPSS. London: Sage.

Figueiredo, B., \& Costa, R. (2008). Estudo de validação da versão portuguesa da Alarm Distress Baby Scale (ADBB). Acta Pediátrica Portuguesa, 39(5), 183-189.

Finger, B., Hans, S.L., Bernstein, V.J., \& Cox, S.M. (2009). Parent relationship quality and infant-mother attachment. Attachment \& Human Development, 11(3), 285-306.

Fuentes, M., Lopes dos Santos, P., Beeghly, M., \& Tronick, E. (2006). More than maternal sensitivity shapes attachment: Infant coping and temperament. Annals of the New York Academy of Sciences, 1094, 292-296.

Goldsmith, H.H., \& Alansky, J.A. (1987). Maternal and infant temperamental predictors of attachment: A meta-analytic review. Journal of Consulting and Clinical Psychology, 55(6), 805-816.

Goldsmith, H.H., Bradshaw, D.L., \& Rieser-Danner, L.A. (1986). Temperamental dimensions as potential developmental influences on attachment. In J.V. Lerner \& R.M. Lerner (Eds.), New directions for child development: Temperament and psychosocial interaction in infancy and childhood (pp. 5-34). San Francisco: Jossey-Bass.

Goldsmith, H.H., \& Campos, J.J. (1982). Toward a theory of infant temperament. In R.N. Emde \& R.J. Harmon (Eds.), The development of attachment and affiliative systems (pp.161-193). New York: Plenum Press.

Goldsmith, H.H., \& Campos, J.J. (1986). Fundamental issues in the study of early temperament: The Denver Twin Temperament Study. In M.E. Lamb, A.L. Brown, \& B. Rogoff (Eds.), Advances in developmental psychology (pp. 231-283). Hillsdale, NJ: Erlbaum.

Grossmann, K., Grossmann, K.E., Spangler, G., Suess, G., \& Unzner, L. (1985). Maternal sensitivity and newborns' orientation responses as related to quality of attachment in Northern Germany. In I. Brether- ton \& E. Waters (Eds.), Growing points in attachment theory and research. Monographs of the Society for Research in Child Development, 50, 233-256.

Guedeney, A., \& Fermanian, J. (2001). A validity and reliability study of assessment and screening for sustained withdrawal reaction in infancy: The Alarm Distress Baby scale. Infant Mental Health Journal, 22(5), 559-575.

Isabella, R.A., Belsky, J., \& von Eye, A. (1989). Origins of infantmother attachment: An examination of interactional synchrony during the infants' first year. Developmental Psychology, 25(1), $12-21$.

Londerville, S., \& Main, M. (1981). Security of attachment, compliance, and maternal training methods in the second year of life. Developmental Psychology, 17, 289-299.

MacKensie, M., \& McDonough, S. (2009). Transactions between perception and reality: Maternal beliefs and infant regulatory behavior. In A. Sameroff (Ed.), The transactional model of development: How children and contexts shape each other (pp. 35-54). Washington, DC: American Psychological Association.

Main, M., Tomasini, L., \& Tolan, W. (1979). Differences among mothers of infants judged to differ in security. Developmental Psychology, $15,472-473$.

Malatesta, C.Z., Culver, C., Tesman, J.R., \& Shepard, B. (1989). The development of emotion expression during the first two years of life. Monographs of the Society for Research in Child Development, 54(1-2), 1-104.

Moran, G., Forbes, L., Evans, E., Tarabulsy, G., \& Madigan, S. (2008). Both maternal sensitivity and atypical maternal behavior independently predict attachment security and disorganization in adolescent mother-infant relationships. Infant Behavior \& Development, 31, $321-325$.

Murray, L., Fiori-Cowley, A., Hooper, R., \& Cooper, P. (1996). The impact of postnatal depression and associated adversity on early motherinfant interactions and later infant outcome. Child Development, 67, 2512-2526.

Murray, L., Stanley, C., Hooper, R., King, F., \& Fiori-Cowley, A. (1996). The role of infant factors in postnatal depression and mother-infant interaction. Developmental Medicine \& Child Neurology, 38(2), 109-119.

Myiake, K., Chen, S.J., \& Campos, J.J. (1985). Infant temperament, mother's mode of interaction, and attachment in Japan: An interim report. In I. Bretherton \& E. Waters (Eds.), Growing points in attachment theory and research. Monographs of the Society for Research in Child Development, 50, 276-297.

Nugent, J.K., Greene, S., Wieczorek-Deering, D., Mazor, K., Hendler, J., \& Bombardier, C. (1993). The cultural context of mother-infant play in the newborn period. In K. MacDonald (Ed.), Parent-child play: Description and implications (pp. 367-389). Albany: State University of New York Press.

Pauli-Pott, U., \& Mertesacker, B. (2009). Affect expression in motherinfant interaction and subsequent attachment development. Infant Behavior \& Development, 32(2), 208-215.

Pawson, R., \& Tilley, N. (1997). Realistic Evaluation. SAGE Publication: Thousand Oaks, CA. 
Roggman, L.A., Langlois, J.H., \& Hubbs-Tait, L. (1987). Mothers, infants, and toys: Social play correlates of attachment. Infant Behavior \& Development, 10, 233-237.

Rothbart, M.K., \& Ahadi, S.A. (1994). Temperament and the development of personality. Journal of Abnormal Psychology, 103(1), 55-66.

Russell, A., Mize, J., \& Bissaker, K. (2002). Parent-child relationships. In P.K. Smith \& C.H. Hart (Eds.), Blackwell handbook of childhood social development (pp. 205-222). Oxford, England: Blackwell.

Sameroff, A. (1975). Transactional models in early social relations. Human Development, 18, 65-79.

Scarr, S., \& Salapatek, P. (1970). Patterns of fear development during infancy. Merrill-Palmer Quarterly, 16, 53-90.

Seifer, R., Schiller, M., Sameroff, A.J., Resnick, S., \& Riordan, K. (1996). Attachment, maternal sensitivity, and infant temperament during the first year of life. Developmental Psychology, 32, 12-25.

Tabachnick, B.G., \& Fidell, L.S. (1996). Using multivariate statistics. New York: HarperCollins.

Tavecchio, L.W.C., \& van IJzendoorn, M.H. (1987). Attachment in social networks: Contributions to the Bowlby-Ainsworth attachment theory. Amsterdam, The Netherlands: North-Holland.

Thompson, R.A., \& Lamb, M.E. (1984). Assessing qualitative dimensions of emotional response in infants: Separation reactions in the strange situation. Infant Behavior \& Development, 7, 423-445. van den Boom, D.C. (1989). Neonatal irritability and the development of attachment. In G.A. Kohnstamm, J.E. Bates, \& M.K. Rothbart (Eds.), Temperament in childhood (pp. 299-318). Chichester, England: Wiley.

van den Boom (1997). Sensitivity and attachment: Next steps for developmentalists. Child Development, 64(4), 592-594.

Veríssimo, M., \& Salvaterra, F. (2006). Maternal secure base scripts and children's attachment security in an adopted sample. Attachment \& Human Development, 8(3), 261-273.

Waters, E., Vaughn, B., \& Egeland, B. (1980). Individual differences in infant-mother attachment relationships at age one: Antecedents in neonatal behavior in a urban, economically disadvantaged sample. Child Development, 51(1), 208-216.

Waters, T. (2004). Learning to love: From your mother's arms to your lover's arms. The Medium, 30(19), 1-4.

Weinfield, N.S., Sroufe, L.A., Egeland, B., \& Carlson, E. (1999). The nature of individual differences in infant-caregiver attachment. In J. Cassidy \& P. Shaver (Eds.), Handbook of attachment: Theory, research, and clinical applications (pp. 64-86). New York: Guilford Press.

Ziv, Y., Aviezer, O., Gini, M., Sagi, A., \& Koren-Karie, N. (2000). Emotional availability in the mother-infant dyad as related to the quality of infant-mother attachment relationship. Attachment \& Human Development, 2(2), 149-169. 